\title{
Somatoforme Störungen
}

\section{Erfolgreich behandeln mit PISO}

- 30\% aller Patienten in der Hausarztpraxis klagen über anhaltende Körperbeschwerden, ohne dass dafür eine organische Ursache gefunden werden kann. Dazu gehören Schmerzen unterschiedlicher Lokalisation, Schwindel und Verdauungsbeschwerden. „Dadurch ist die Lebensqualität häufig stark beeinträchtigt, gleichzeitig verursachen diese $\mathrm{Pa}$ tienten hohe Kosten im Gesundheitssystem durch fehlindizierte diagnostische und therapeutische Maßnahmen“, sagte Prof. Peter Henningsen, München. Die Beschwerden schränken diese Menschen im Alltag stark ein und bergen das Risiko für eine Depression bzw. einen sozialen Rückzug.

Für solche somatoformen Störungen sind in den letzten Jahren neue effiziente psychosomatische Therapieansätze entwickelt worden.

\section{Psychodynamisch interpersonelle Therapie}

Dass eine spezielle Psychotherapie bei diesen Patienten erfolgreich ist, zeigte die PISO-Studie. PISO steht für „psychodynamisch interpersonelle Therapie bei somatoformen Störungen“. Die in die Studie eingeschlossenen 208 Patienten litten seit durchschnittlich zehn Jahren an unterschiedlichsten Symptomen wie Schmerzen, Schwindel, Müdigkeit oder Reizdarm. Die Hälfte dieser Patienten wurde gemäß den aktuellen Leitlinien für somatoforme Probleme ausführlich informiert und beraten. Die übrigen unterzogen sich der sog. psychodynamisch interpersonellen Therapie, d. h. in 12-wöchentlichen Sitzungen klärten Psychotherapeuten die Patienten über die Hintergründe psychosomatischer Erkrankungen auf, schulten sie im Um- gang mit ihren Beschwerden und zeigten ihnen Entspannungstechniken. „Während die Entwicklung in der konventionell behandelten Gruppe bald stehen blieb, besserten sich die Symptome und die Lebensqualität der übrigen $\mathrm{Pa}$ tienten auch noch neun Monate nach der Therapie“, so Henningsen. Auch sank der Anteil der Teilnehmer mit einer schweren Depression in der PISOGruppe von 44 auf 26\%. Auch im weiteren Verlauf ging die Zahl der Arztbesuche deutlich zurück. „Selbst Patienten mit schweren somatoformen Störungen und starker Depressivität können mit dieser Therapie erfolgreich behandelt werden“, so das Fazit von Henningsen.

Dr. med. Peter Stiefelhagen .

- Quelle: Deutscher Kongress für Psychosomatische Medizin und Psychotherapie, 23.--26. März 2011 in Essen.

\section{Fibromyalgie}

\section{Eine Störung der Stressverarbeitung?}

- Das Fibromyalgie-Syndrom ist ein klinischer Beschwerdekomplex, bei dem multilokuläre Schmerzen in unterschiedlichen Körperbereichen im Vordergrund stehen und keine ursächlich erklärenden strukturellen Schädigungen oder biochemischen Abweichungen von rheumatologischer, orthopädischer oder neurologischer Seite diagnostiziert werden können. Die Frage, ob es sich bei der Fibromyalgie aber doch um eine rheumatische Erkrankung handelt, wird kontrovers diskutiert.

In den letzten Jahren wurden mithilfe moderner bildgebender Verfahren neue Erkenntnisse gewonnen, die wie Mosaiksteine ein zunehmend klares pathogenetisches Bild der Erkrankung liefern. „Die Befunde sprechen dafür, dass es sich bei der Fibromyalgie um eine Störung der Stressverarbeitung handelt, bei

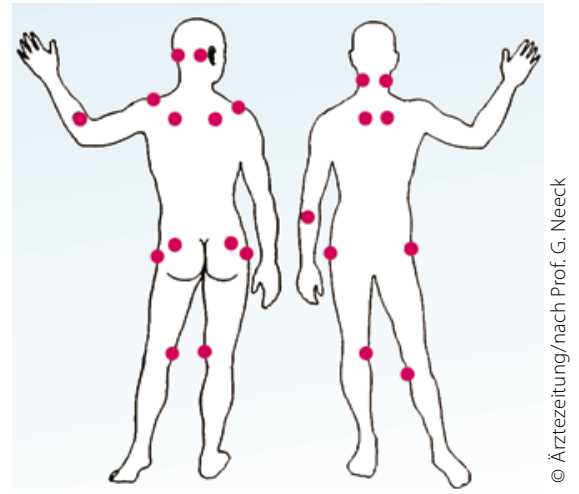

Typische Druckpunkte bei Fibromyalgie: Tenderpoints.

der zentralen Prozessen in den dafür zuständigen Bereichen des Gehirns eine wesentliche Bedeutung zukommt", so Prof. Ulrich T. Egle, Gengenbach. Sowohl biologische als auch psychosoziale Stessoren fungieren als Auslöser. Un- günstige Umweltbedingungen in der Kindheit erhöhten die Vulnerabilität für die Manifestation der Fibromyalgie. Nach Einsetzen der Beschwerden wirken iatrogene, familiäre und intraindividuelle Einflussfaktoren chronifizierend.

Eine wesentliche Aufgabe des Hausarztes ist es, den betroffenen Patienten umfassend über diese biopsychosozialen Zusammenhänge zu informieren. „Erklären Sie dem Patienten, dass der Erkrankung ein Stressverarbeitungsproblem zugrunde liegt“, so Egle. Dies ermögliche dem Patienten, bei der Therapie aktiv mitzuarbeiten. Auch solle man bedenken, dass wiederholte Aufnahmen in rheumatologischen Fachkliniken die Chronifizierung der Beschwerden nur begünstigten.

STI =

- Praxis Update Allgemeinmedizin, Berlin, 6.-7.5.2011 